\title{
A link-based visual search engine for Wikipedia
}

\author{
David Milne \\ Department of Computer Science, University of Waikato \\ Private Bag 3105, Hamilton, New Zealand \\ +6478384021 \\ d.n.milne@gmail.com
}

\begin{abstract}
This paper introduces Hōpara, a new search engine that aims to make Wikipedia easier to explore. It works on top of the encyclopedia's existing link structure, abstracting away from document content and allowing users to navigate the resource at a higher level. It utilizes semantic relatedness measures to emphasize articles and connections that are most likely to be of interest, visualization to expose the structure of how the available information is organized, and lightweight information extraction to explain itself.

Hōpara is evaluated in a formal user study, where it is compared against a baseline system that provides the same functionality without visualization, and the incumbent Wikipedia search interface. Although objective performance measures are inconclusive, subjective preference for the new search engine is very high. All but one of twelve participants preferred the new system over Wikipedia, despite the latter's familiarity. The visualization component was a deciding factor: all but two participants chose it over the non-visual system.
\end{abstract}

\section{Categories and Subject Descriptors}

H.5.4 [Information Interfaces and Presentation]: Hypertext/Hypermedia - navigation, user issues.

\section{General Terms}

Experimentation, Human Factors.

\section{Keywords}

Exploratory Search, Information Visualization, Information Retrieval, Wikipedia, Semantic Relatedness.

\section{INTRODUCTION}

Anyone who has browsed Wikipedia has likely experienced the feeling of being happily lost, browsing from one interesting topic to the next and encountering information that they would never have searched for explicitly. Wikipedia's articles are interlaced by a gigantic network of internal links. Anywhere you go, there are attractive, even seductive options to go elsewhere.

Are Wikipedia's links too much of a good thing? As Figure 1 illustrates, the abundance of paths can cause distraction and disorientation. Here the cartoonist from the online web comic xkcd enters Wikipedia with specific information need: he wants to find out about the Tacoma Narrows Bridge, which famously twisted itself apart in mild winds in 1940. His forays are initially

This paper was published in the proceedings of the New Zealand Computer Science Research Student Conference (NZCSRSC) 2010. Copyright is held by the author/owner(s). NZCSRSC 2010, April 2010, Wellington, New Zealand focused and relevant, but soon degrade into three wasted hours (at least in regards to the initial information need) of aimless wandering [14].

Hypertext has long been championed as a way to improve navigation in large document collections. Wikipedia represents a preview of what large corpora would look like if automatic hypertext generation [1,6] were able to match the accuracy of humans and gained widespread acceptance. It is extraordinarily large and densely interlinked, and almost every link has been constructed and vetted manually. Yet information seekers still have difficulty navigating the resource. Scale introduces new challenges. At some point a hypertext system grows to such an extent that links alone are not enough to navigate the documents; something more is needed to navigate the links [11].

With this challenge in mind, we introduce Hōpara: a new search engine that aims to make Wikipedia and its underlying link structure easier to explore. Before describing the details of the system, we first survey the related work that surrounds it. This includes information visualization, and specifically visualization of Wikipedia. Sections 3 and 4 describe Hōpara's interface and the algorithms behind it, respectively. The system is evaluated with a formal user study in Section 5. Section 6 discusses the implications and limitations of the experiment, and sketches out our plan for the near future.

\section{RELATED WORK}

Over the course of human evolution, our perceptions have been gradually honed and attuned to deal with the spatial-visual arrangement of objects. We have specialized in using vision to navigate the world. It follows that retrieval systems should allow us to apply our visual skills to navigate virtual spaces.

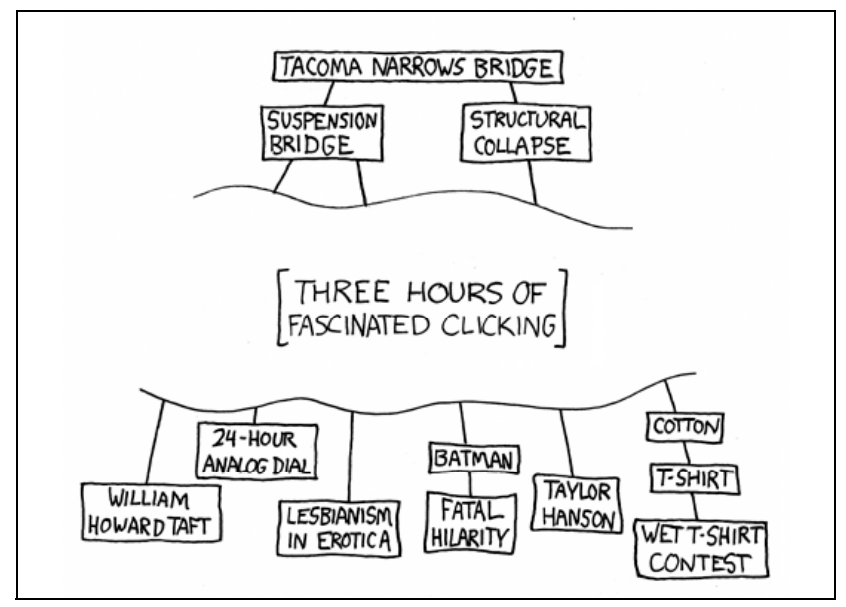

Figure 1: The problem with Wikipedia 


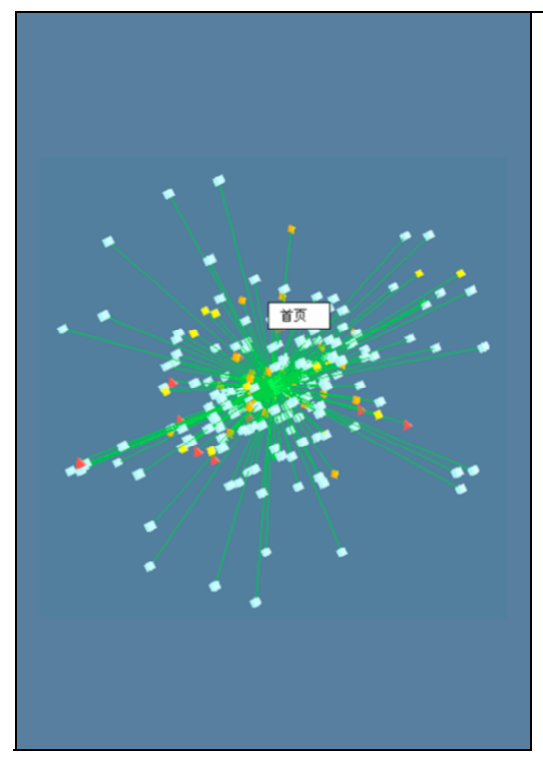

Figure 2: A WikiVis visualization

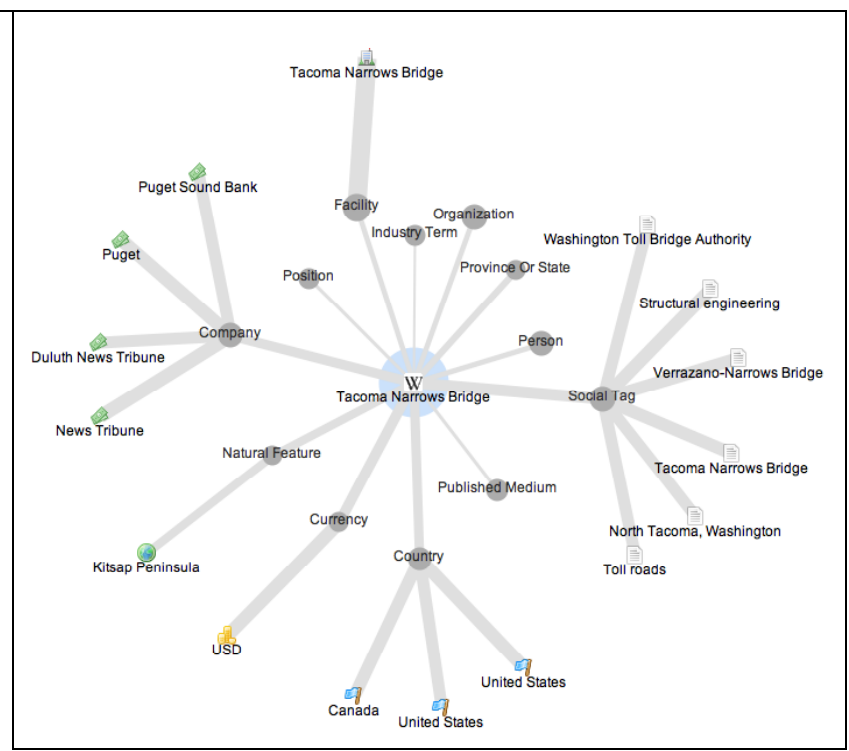

Figure 3: A ThinkPedia visualization of topics related to Tacoma Narrows Bridge \begin{tabular}{|l} 
Tacoma Narrows Bridge \\
Social Tag \\
Washington Toll Bridge Authority \\
Structural engineering \\
Verrazano-Narrows Bridge \\
Tacoma Narrows Bridge \\
North Tacoma, Washington \\
Toll roads \\
Country \\
Canada \\
United States \\
United States \\
Currency \\
USD \\
Natural Feature \\
Kitsap Peninsula \\
Company \\
Puget Sound Bank \\
Puget \\
Duluth News Tribune \\
News Tribune \\
Facility \\
Tacoma Narrows Bridge \\
\end{tabular}

Figure 4: An alternative interface for ThinkPedia
Unfortunately, attempts to apply information visualization to information retrieval have met with limited success [7]. Chen and $\mathrm{Yu}$ [3] conducted a meta-analysis of 35 information visualization studies published between 1991 and 2000. At the time they were only able to locate six studies that satisfied the criteria for analysis and direct comparison-e.g., task type, use of spatial-visual interfaces, comparison to control. Broadly, their conclusions based on the six studies were:

- The cognitive abilities of participants had a greater effect than the choice of interface (visual vs. control).

- Simple, lightweight visualizations were more effective than complex ones.

- The combined effect of visualization was not statistically significant (due in part to the small sample size).

This does not mean that information visualization is not useful. It has been applied very successfully to information analysis, to expose and communicate general trends, patterns and outliers in data [4]. The point is that its applications for retrieval are less obvious. Searching is typically a short-term, secondary activityone searches for something in order to do something else. There is little room for deep, complex interaction unless one supports broader information seeking and exploration, which is inherently complex and difficult to cater for. Additionally, the data involved is almost exclusively text, which does not lend itself well to visualization unless coupled with something else-dates, quantities, faces and places - or has some quantitative propertysignificance, popularity, similarity-that can be conveyed spatially. Thus effective visualization techniques for general, open-domain text retrieval remain elusive.

Wikipedia has received a considerable amount of interest from visualization researchers. This is not surprising; it is large, easily accessible, and has many interesting properties to visualize. Holloway et al. [10] investigate the semantic coverage and bias of Wikipedia by visualizing its categories and authors. Chris Harrison has produced several (unpublished) visualizations, including timelines of article popularity ${ }^{1}$ and graphs of categories and their connections. ${ }^{2}$ Bruce Herr, Todd Holloway, and Katy Borner represent Wikipedia's topic coverage as large mosaics. ${ }^{3}$

Visualization of Wikipedia's edit history-the flow of contributions, revisions and reverts that make up each collaboratively produced article-has proved extremely fruitful. History flows [16], tilebars [5], chromograms [17] and timelines [15] have all been used to expose vandalized, contentious or under-developed articles, and assist with conflict resolution.

The examples above support analysis: they help researchers and editors understand broad trends in the content and development of Wikipedia. Mirroring the imbalance of the field in general described previously, visualization of Wikipedia for the purposes of retrieval has been much less fruitful. To our knowledge there are only two relevant studies. The first, by Biuk-Aghai [2], experiments with various layouts for visualizing articles and the connections between them as 3D graphs. Unfortunately the resulting system-WikiVis - received no evaluation. Anecdotally, its visualizations (Figure 2 is a typical example) are illegible at first glance, and their utility for searching is questionable.

The second relevant system, developed by Hirsch et al [8], is ThinkPedia. Figure 3 shows the visual component of this system. Not shown is a search box (in which the user has entered Tacoma Narrows Bridge), a list of closely related queries (e.g. Tacoma Narrows Bridge Collapse), and a frame containing the entirety of the relevant Wikipedia article. The interface uses the professional visualization toolkit ThinkMap, ${ }^{4}$ and is consequently cleaner and more legible than WikiVis. Nodes and relations are clearly labeled and can be readily identified. Relations are grouped so that similar

\footnotetext{
${ }^{1}$ http://www.chrisharrison.net/projects/wikitop

${ }^{2}$ http://www.chrisharrison.net/projects/clusterball

${ }^{3}$ http://www.scimaps.org/maps/wikipedia

${ }^{4}$ http://thinkmap.com
} 


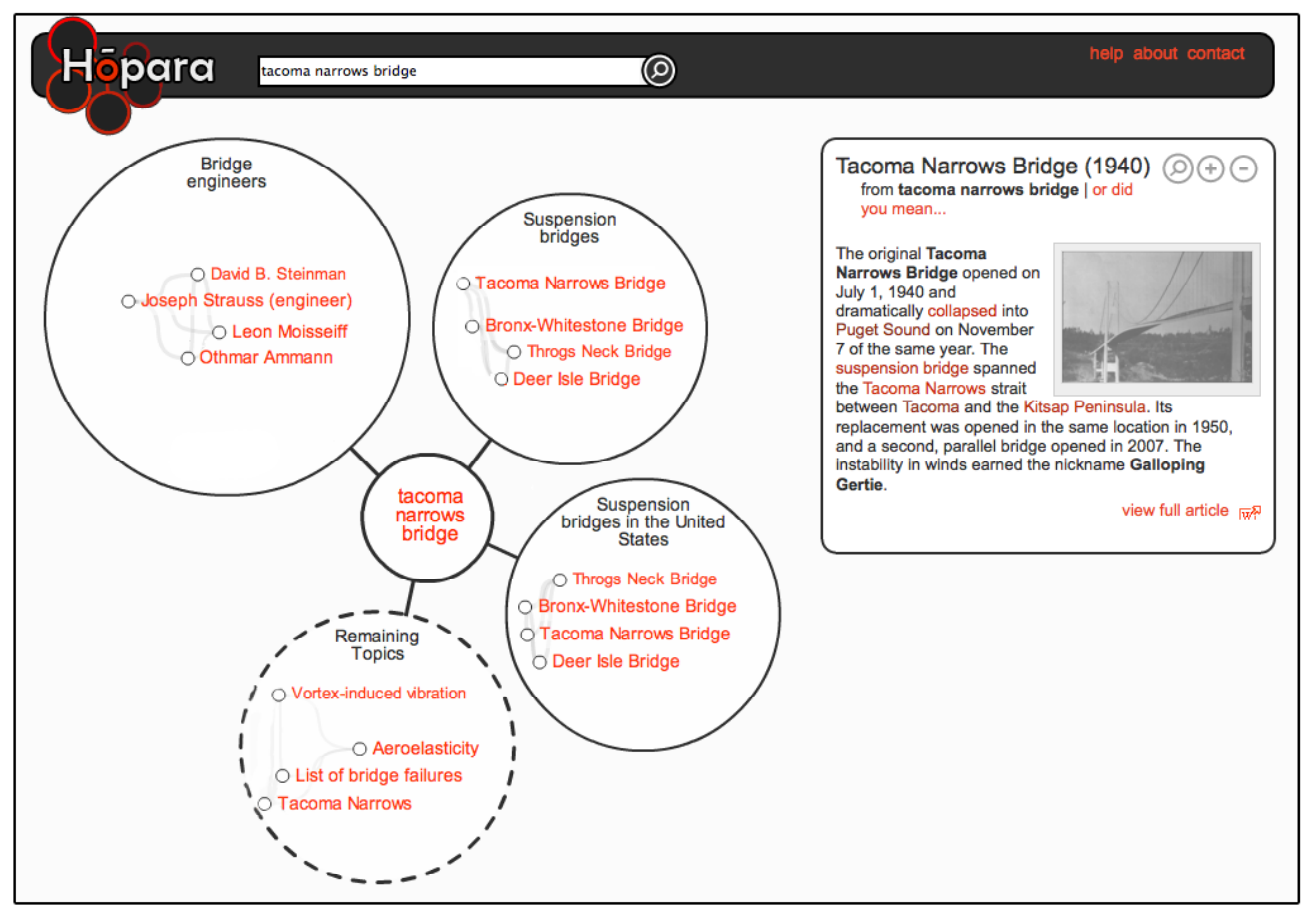

Figure 5: The Hōpara search engine, showing topics related to tacoma narrows bridge

topics (e.g. the companies Puget Sound Bank and Duluth News Tribune) are collocated spatially.

However, the same properties-legibility and spatial organization - can be achieved trivially without visualization. Figure 4 communicates the same information as Figure 3. It seems reasonable to expect the visualization to convey something more, as payoff for the unfamiliarity and technical difficulties it causes - additional software requirements, browser navigation and bookmarking issues, etc. The visualization is also much less space-efficient than the list-based alternative, and scales less easily. A slider is provided to expand the graph (to see more of the Company, Person or Facility groups, etc), but this causes layout issues (overlapping labels and edges) and necessitates panning. Additionally, many of the suggestions ThinkPedia makes are of questionable use. Some are irrelevant (e.g. USD has no particular significance, and Puget links to a French commune). Others are redundant (e.g. United States is listed twice and is extremely general, Tacoma Narrows Bridge is listed three times) or incomplete (relevant natural features such as the Tacoma Narrows Straight are not listed, even when the graph is fully expanded).

Overall, one wonders how useful the visualization and the connections it expresses really are. There has not been a formal evaluation to investigate this. In anecdotal studies, users found the tool to be "beautiful" and "fun", but also "disordered" and "frustrating" to search with [8]. The developers have had more success in constructing similar interfaces to more structured resources, such as the semantic database Freebase and the corporate wiki Confluence [9].

The primary metaphor for both WikiVis and ThinkPedia is to visualize a subset of Wikipedia as a graph, where the nodes are concepts and the edges between them indicate relatedness. Both systems must draw connections between articles, and one might reasonably expect them to use inter-article hyperlinks to do so.
Instead, the manually defined links are abandoned in favor of automatically generated connections. WikiVis uses co-authorship analysis to identify related articles, while ThinkPedia uses the SemanticProxy web service. ${ }^{5}$ One wonders why, given that Wikipedia provides connections that have been handcrafted to help readers navigate from one article to the next.

\section{HŌPARA}

The Māori who traversed the Pacific Ocean to discover New Zealand were skilled navigators, and hōpara is their word for exploration. The Hōpara system, shown in Figure 5, is a new search engine that applies semantic relatedness, information extraction and visualization to make Wikipedia easier to explore. ${ }^{6}$

The upper area is a classic search box where the user has entered Tacoma Narrows Bridge; the cartoonist's query from Figure 1. Below and to the left of the search box is a visualization of the related topics: the engineers involved, other similar bridges, and some of the engineering concepts connected to its demise. To the right is an extract of the relevant article.

The query is ambiguous: Wikipedia contains an article about the bridge that famously collapsed in 1940, and another about its replacement. When queries have multiple interpretations, the system selects one sense automatically but makes the remaining senses available with the link entitled or did you mean. The system also allows for synonymy: e.g. the query galloping gertie (the bridge's nickname) takes the user to the same result. The process by which senses and synonyms are identified and resolved is described in Section 4.

\footnotetext{
${ }^{5}$ http://semanticproxy.com

${ }^{6}$ http://www.nzdl.org/hopara
} 


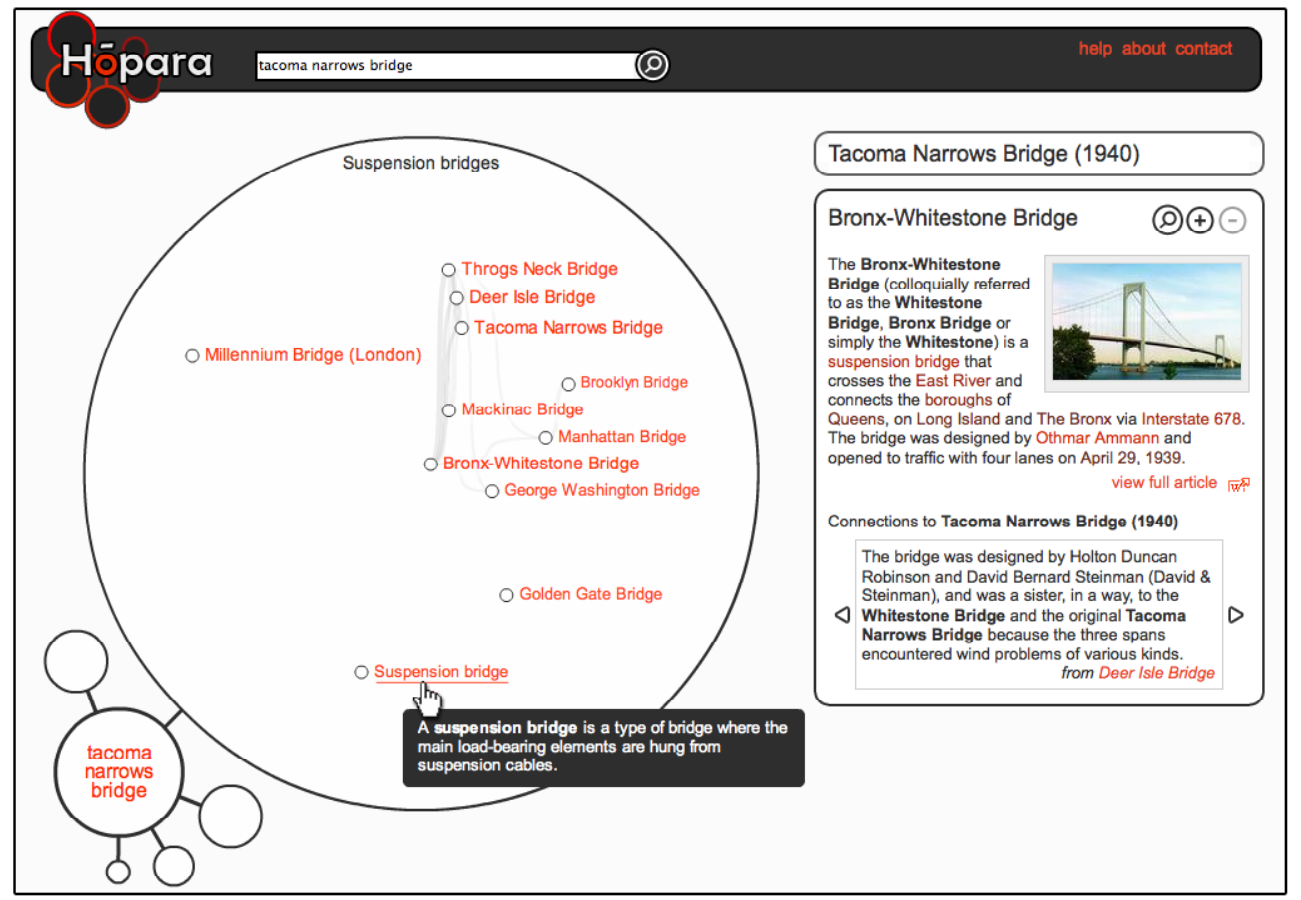

Figure 6: The Hōpara search engine, focusing on Suspension bridges (specifically the Bronx-Whitestone Bridge)

The visualization on the left of the interface displays the user's query in the center, and four categories or groupings of suggestions surrounding it. Within each grouping is a small graph whose nodes are topics and edges are semantic relations between them. Larger topics, such as Leon Moisseif (the project's lead engineer) are more strongly related to the query. An edge between two topics indicates that they are semantically related to each other, and thicker edges indicate stronger relations.

Only four groupings of topics can be shown at a time. Moving clockwise from the top left corner of Figure 5, the first three represent the categories containing the strongest, most relevant suggestions. Their size represents their expected value to the searcher. The fourth grouping, indicated with a dotted outline, shows the best topics that did not belong to the top categories. If this is clicked, the visualization smoothly rotates to reveal the remaining, less relevant categories. Thus the system can scale to show many categories of topics, without panning or zooming.

Similarly, only the four best topics are shown within each grouping. If more are available, then scalability is achieved by allowing categories to be expanded. If the user clicks Suspension Bridges in Figure 5, for example, then system smoothly transitions to the layout shown in Figure 6. Animation is used carefully to minimize disorientation: the desired category slickly expands and the others shrink and move out of the way. More nodes are added to the graph, which is smoothly but quickly rearranged using a force-directed layout that encourages related topics to be clustered together spatially, and others (such as the general topic Suspension Bridge, or Millennium Bridge - the only one not located in the U.S.) to be separated.

Mousing over any topic link reveals a tooltip containing the first sentence of the article in question, as shown for Suspension Bridge in Figure 6. The user can click on any topic to open a box on the right side of the interface, as shown for Tacoma Narrows Bridge (1940) in Figure 5 and Bronx-Whitestone Bridge in Figure
6. The box contains the first paragraph and an image extracted from the article, and a link to Wikipedia. It also contains sentence snippets to explain how the topic relates to the original query. In Figure 6 this reveals that the Bronx-Whitestone Bridge and the Deer Isle Bridge suffered similar design problems as Galloping Gertie, which explains why Hōpara emphasizes them over the other bridges. The methods for judging the relevance of suggestions and extracting the snippets to explain them are described in Section 4.

On the top right corner of the Bronx-Whitestone Bridge topic box in Figure 6 is a set of three buttons that control how it can be investigated further. The first explores it as a new query. The second adds it to the current query, to explore things that relate to or connect both bridges. The third button removes it from the query-it is disabled in Figure 6 because Bronx-Whitestone Bridge is not part of the current search.

Multi-topic queries can also be built directly in the search box. For example, the query bridge failure is automatically recognized as two distinct topics: Bridge and Structural failure. The interaction is much the same as for the mono-topic query in Figures 5 and 6, except that the visualization on the left is narrowed to contain only suggestions that relate to (or bridge between) both query topics (e.g. Catastrophic failure, Structural design), and multiple connection snippets are shown within each topic box (because there are multiple query topics to connect to).

Care has been taken to minimize the negatives introduced by the system's technical requirements. Most of the interface is built using standard HTML elements under the AJAX framework. The visualization itself is implemented using the Prefuse Flare toolkit, ${ }^{7}$ and is seamlessly integrated into the page. It requires only a browser with JavaScript and Flash enabled (both are almost

\footnotetext{
${ }^{7}$ http://flare.prefuse.org
} 


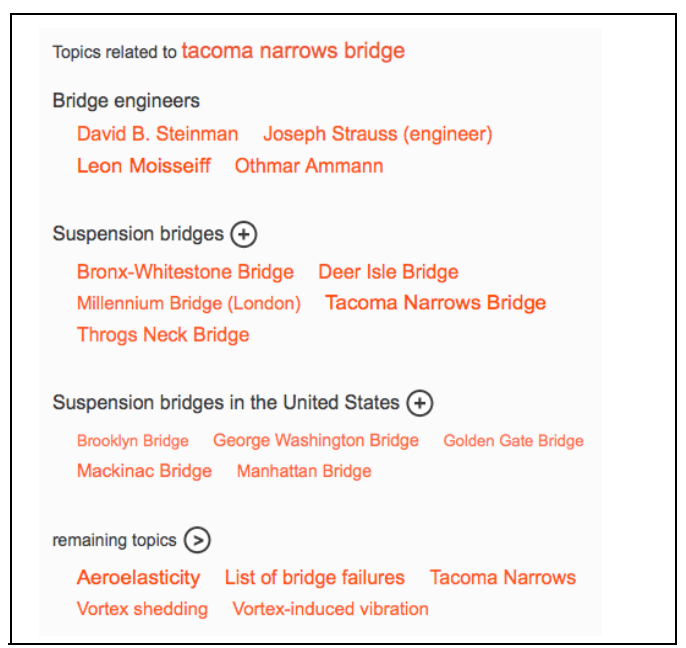

Figure 7: Tag-based alternative to visualization

ubiquitous). Unlike many similar systems, browser navigation (history, bookmarking, etc.) is preserved.

\section{MINING WIKIPEDIA'S LINKS}

The backend of Hōpara is built upon the Wikipedia Miner toolkit, a Java-based API and suite of web services for navigating and making use of the structure and content of Wikipedia. ${ }^{8}$ This provides much of the functionality that Hōpara depends on, including matching search terms to articles, gathering related topics, grouping these suggestions into categories, and explaining connections between topics.

Searching is provided by Wikipedia Miner's indexing of article titles, redirects and anchor texts found within inter-article links. Anchors are particularly useful because they encode both synonymy and ambiguity. For example, Hōpara knows that Galloping Gertie and Original Tacoma Narrows Bridge are synonymous because both are used as anchors to the same article. It also knows that Tacoma Narrows Bridge is ambiguous and which sense is most likely of interest, because the anchors containing this phrase go to two different locations; $43 \%$ to Galloping Gertie and 57\% to its replacement. Resolving queries is a matter of checking n-grams against the anchor vocabulary.

To suggest related topics, Hōpara gathers all articles that link to the query articles or are linked by them. The resulting topics are ranked by how strongly they relate to all query topics using the WLM measure [13]. Only those that are sufficiently related are used, to avoid sending the user off topic. WLM measures the semantic similarity of two Wikipedia articles by comparing their incoming and outgoing links. The formula is:

$$
\text { relatedness }(a, b)=\frac{\log (\max (|A|,|,| \mid))-\log (|A \cap B|)}{\log (|W|)-\log (\min (|A|,|B|))}
$$

where $a$ and $b$ are the two articles of interest, $A$ and $B$ are the sets of all articles that link to $a$ and $b$ respectively, and $W$ is set of all articles in Wikipedia. This probably sounds expensive and slowlinks to and from query topics are gathered as suggestions, and links to these are in turn used for ranking - but the process is extremely efficient in practice. Approximately 11,000 relatedness measures can be made per second on a $3 \mathrm{GHz}$ machine.

\footnotetext{
${ }^{8}$ http://wikipedia-miner.sf.net
}

\section{Figure 8: Exploratory search tasks}

Having gathered and ranked the suggested topics, the next task is to organize them. Each article in Wikipedia is manually tagged with one or more categories. Hōpara gathers the relevant categories for the suggestions and ranks them by the total strength (relatedness to query topics) of the top five suggestions within them. Categories containing fewer than three suggestions are discarded, and the remaining uncategorized suggestions form a Miscellaneous group, which is presented last.

To explain connections between two topics, say $a$ and $b$, Hōpara gathers sentences in $a$ that mention $b$, sentences in $b$ that mention $a$, and sentences in other articles that mention both. A fortunate side-effect of the WLM measure is that all the articles that could possibly contain these sentences are known in advance: this is the set $A \cap B$ in the relatedness formula above. The remaining work of detecting topic mentions within the articles and identifying sentence boundaries around them is done on the fly.

\section{EVALUATION}

We undertook a user study that evaluates Hōpara and its underlying algorithms for their ability to facilitate exploratory search. The study compares three systems: the incumbent Wikipedia interface, the full Hoppara system described in the previous section, and a baseline system that packages the same functionality in a more familiar interface.

Wikipedia's support for exploration is already better than most information sources. At the time of writing it provides extensive inter-article links, portals to organize articles thematically, a category network to organize them hierarchically, and an autocomplete system to suggest queries as they are typed. It represents a robust, challenging baseline.

As explained in Section 2, information visualization for the purposes of retrieval has had limited success in the past. It is easy to construct visualizations that do more harm than good. To be successful, they have to provide some kind of payoff while minimizing the increased complexity and unfamiliarity they cause. To isolate the effect of Hōpara's visualization, we constructed a baseline system that is identical in every way, except that the graphs of related topics (the circular categories) are replaced with the tag clouds shown in Figure 7. Comparison with this baseline provides direct insights into whether the visualization provides a positive difference. 


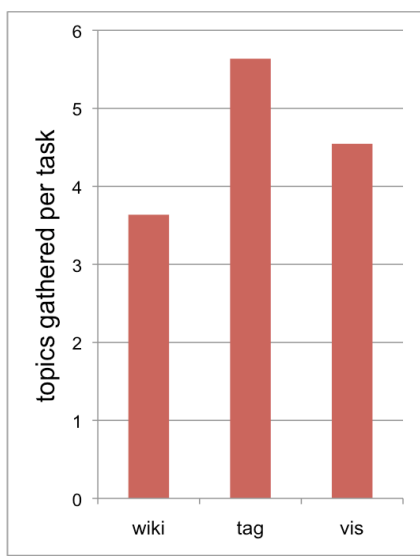

a)

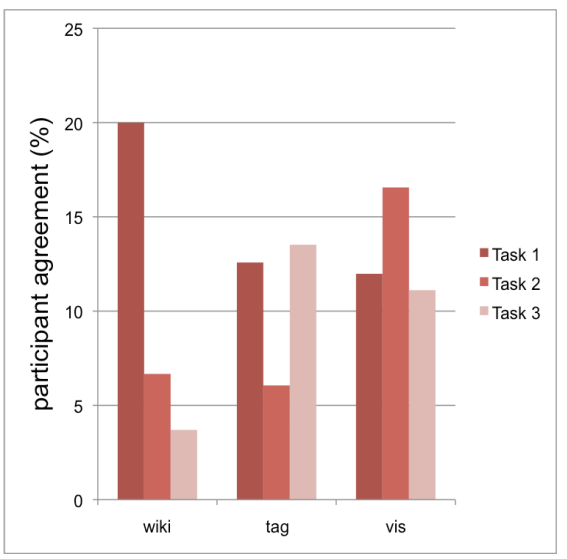

b)

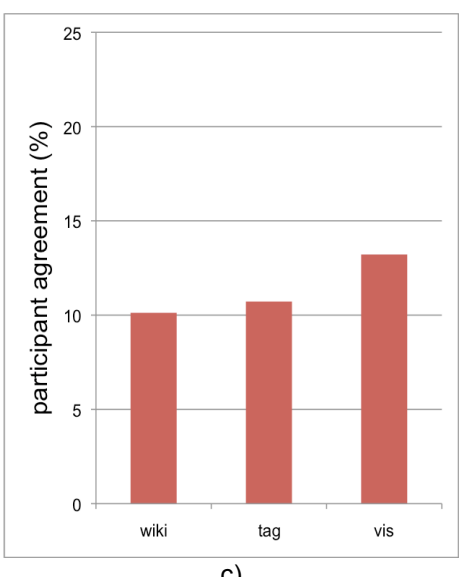

c)

Figure 9: Performance of tasks

For the remainder of this section the three systems-Wikipedia, Hōpara, and the tag-cloud baseline-will be referred to as wiki, vis and tag respectively.

\subsection{Subjects and Tasks}

Twelve participants were observed as they interacted with the three systems. All were experienced searchers participating in a graduate level computer science course. All but one was a regular visitor to Wikipedia. Sessions typically lasted for one and a half hours, and were conducted in a controlled environment with video and audio recording and an observer present. Data was also collected from questionnaires and system logs.

Each user performed the three tasks shown in Figure 8 by gathering the Wikipedia articles they felt were relevant. The tasks were modeled after [12] to be open-ended, multifaceted, and provide imaginative context for the participant to relate to. Performing a task amounted to using one of the systems, building a list of relevant article titles, and supporting each selected article with a short sentence (either copied from the system or typed freely) to explain its relevance. Each task was performed on a different system and the order in which the systems were used was staggered to counter the effects of bias and transfer learning. Participants were given as much time as they needed to familiarize themselves with the system (typically 5-10 minutes) before being given a task, but were specifically asked to spend close to 15 minutes performing each one.

\subsection{Results}

This section compares the three systems - wiki, tag and vis-on the basis of objective measures of task performance and subjective impressions gathered from questionnaires.

\subsubsection{Task Performance}

One limitation of this study (and other investigations of exploratory search) is that we could not determine the answers to the tasks - which are deliberately ambiguous and open-ended - in advance. Therefore there is no ground truth against which to judge the lists of topics by each participant.

A simple measure of task success is the number of topics gathered. A good system should allow participants to locate more relevant topics in the same time. Figure 9a shows that users gathered more topics with the tag and vis systems than wiki, indicating that in this respect Hoppara is an improvement. However, the numbers are quite low across all systems.
Another measure is the extent to which participants agree with each other. Complete agreement is extremely unlikely because the tasks are open-ended and participants have limited time to follow the available leads. Nevertheless, a recommendation is more likely to be correct if multiple participants agree on it. It would not be fair to measure agreement across systems, because two of them (tag and vis) return the same results and would consequently gain an unfair advantage. Instead Figure $9 \mathrm{~b}$ measures the agreement of participants within each combination of task and system. The first column, which measures the agreement between the four participants who used wiki to perform the Walking around the South Island task, is a clear outlier. The other two tasks were performed much more poorly with this system. This is because Wikipedia contains a couple of articles (Tramping in New Zealand and New Zealand tramping tracks) that address the first task directly by organizing lists of links to all of the relevant articles. Answers to the other tasks are scattered throughout Wikipedia with no meta-articles to organize them, and were therefore significantly more difficult to answer. The other two systems are able to answer tasks relatively consistently, whether relevant meta-articles were available or not.

Figure 9c averages agreement measures across tasks to provide a single measure per system. It identifies vis as the system for which participants were most consistent with each other.

\subsubsection{Questionnaire Responses}

Each participant was given four questionnaires to record their subjective impressions of the systems. Three post-task questionnaires were used to capture usability issues, most of which were minor and will not be discussed in this paper. Of more interest is the final questionnaire, in which participants compared the three systems directly according to various criteria such as quality of search results and ease of navigation. The ratings were given using the triangles shown in Figure 10, which allow all three systems to be compared simultaneously. For example, a mark in the center indicates that all three systems are equivalent. A mark at the top corner indicates a strong preference for wiki, and half way between top and center indicates a moderate preference. A mark below the center rates wiki as worse than the other two. Each dot within each triangle in Figure 10 is the rating made by a single participant. The larger circle represents the average rating for all participants. Each rating was explained and justified by participants either orally or by typing free text. 


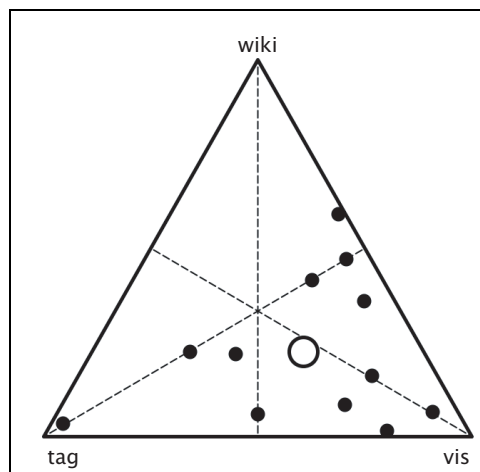

a) How easy was it to complete the tasks with each system? Did one make them particularly easy?

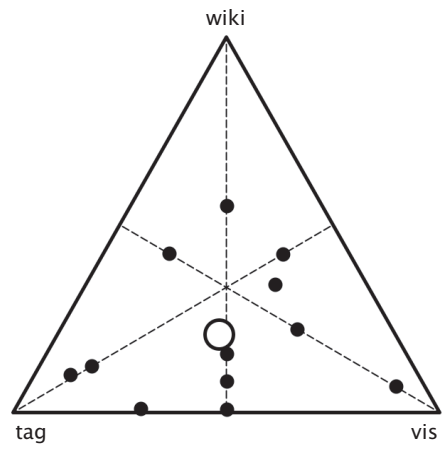

d) Did the systems provide good search results? Did one system provide better results than another?

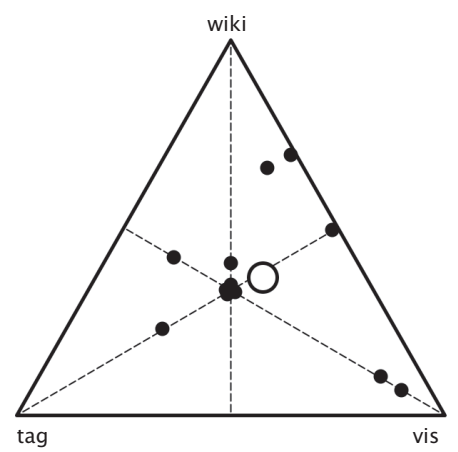

b) How easy was each system to learn how to use? Was one particularly straight-forward?

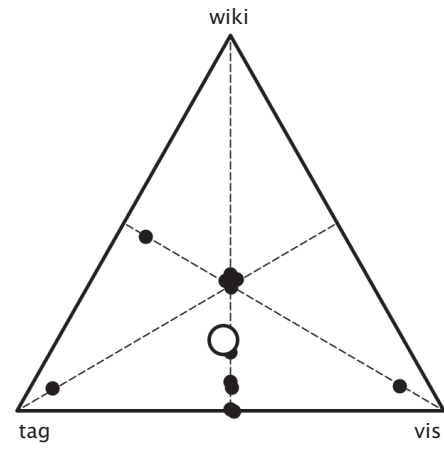

e) Did the systems provide useful links to follow? Did one system provide better links than another?

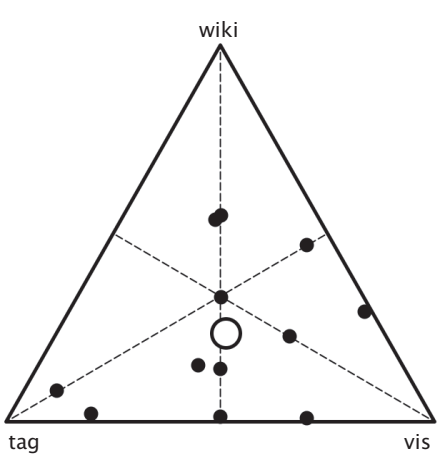

c) How easy was it to navigate the systems? Was one particularly easy to find your way around?

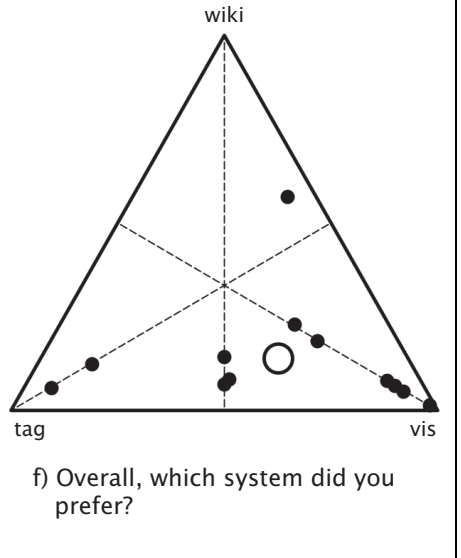

Figure 10: Subjective comparisons of the wiki, tag and vis systems

The first question (Figure 10a) asks whether one of the systems made tasks particularly easy to complete. Most of the responses are scattered towards the vis system.

It was easiest with vis, 2nd was tag, It wasn't very easy with Wikipedia. When I type in the query [in tag or vis], it takes the quote out of the article to show how it is related to the query. [In wiki] if I wanted that, I would have to do a ctrl-f and search for the words I am looking for. Between vis and tag, the circles just made everything clearer.

Figure $10 \mathrm{~b}$ asks whether one system was particularly easy to learn. Half the responses are clustered around the center of the triangle, indicating that there was little difference between the systems. Two participants chose Wikipedia as easier to learn, and cited its familiarity and similarity with traditional search engines and web pages. Another two participants had a strong preference for the vis system, because they felt the visualization more clearly communicated how the available information was organized and connected.

They are all very easy to use.

I think wiki is easier to learn, as I said it is familiar to me. More like other web search.

One advantage of viz is that it gives a clearer picture of how things are related.
Participants had strong but conflicting preferences when asked whether one system was easier to navigate than the others (Figure 10c). Two participants chose the wiki system, again because of its simplicity and familiarity. Others chose against it, and felt that the links they needed to follow were hidden within lengthy prose. The tooltips and connection snippets provided by tag and vis made it easier for participants to judge the relevance of links. Those who chose against vis pointed out that it does not clearly indicate when categories can or cannot be expanded, or how many categories are available.

Wiki has a classic search box. Most people know how to use that.

Wikipedia wasn't very easy to navigate around. I couldn't tell which of the links were useful or not. I had to click on them and go there and have a read, then go back and everything. But with vis and tag I could tell.

The connections snippets made it a bit clearer the effect of following that link.

Figure 10d asks whether one system provided better search results than another. Three participants chose wiki, because it gave them more flexibility in how queries were specified. In tag and vis, query terms must be matched to anchor texts. This forced users to think carefully about which keywords to use, and sometimes made for brittle searching; slight variations in spelling or pluralism could have a large effect on the results. Additionally, both tag and 
vis did not behave as users expected when queries involved classes of topics, e.g. the query jazz musicians returns topics that are related to both Jazz and Musician but are not Jazz Musicians.

Hard to say. I think wiki provides better results. Depends on whether I clearly know the keyword to use. Most of the time when we search for stuff, we don't know.

Figure 10e asks about the usefulness of the links provided by the three systems. One third of participants felt there was no significant difference between them. The remaining participants chose against wiki, again citing that the links they needed were hidden within lengthy articles. They also appreciated the way tag and vis organized the suggested links into categories, and explained them with sentence snippets.

I could easily spend all day following links on all the systems. The categorisation of links for tag and vis allowed me to cut the chaff from the wheat easily.

[I choose tag and vis], just because it is easy to see the connection. I don't have to read [laugh] or think more.

Finally, Figure 10f asks which system was better overall. Only one participant chose wiki-they felt that some of the features in tag and vis were too unfamiliar and awkward to use. Of the remaining participants, only two chose tag over vis-they liked Hōpara's functionality, but preferred it when packaged in a more traditional interface. Overall, $3 / 4$ of participants indicated that vis was at least as good as the other two systems, and $1 / 2$ identified it as the best system. The results are clear-cut: the combination of Hōpara's functionality and visual interface is a winning one.

\section{DISCUSSION AND CONCLUSIONS}

This paper has introduced Hōpara, a new search engine that harnesses and visualizes the links within Wikipedia to make the resource easier to explore. The system offers several advances over previous visualizations of Wikipedia. It directly uses the links and categories that have been handcrafted into the resource to support navigation, as they were originally intended. It ranks the suggestions it provides to emphasize those that are most likely to be of interest, and explains their relevance in plain English. It illustrates how the available information is organized, using spatial and visual cues that cannot easily be replicated without the use of visualization. It scales gracefully when the number of suggestions available would otherwise be overwhelming and disorientating.

A user study has been conducted, in which participants felt strongly that Hōpara was an improvement over the incumbent interface to Wikipedia and that its visualization component offered concrete advantages over the more traditional, simpler alternative. This evaluation, despite being more rigorous than other similar studies, is still a weak point. We were not able to provide conclusive, objective proof of Hōpara's utility; only of users' subjective feelings about it. In the near future we plan to conduct longer-term ethnographic studies, where participants are not given artificial tasks but instead have their own reasons to use the system. This should provide deeper insights into how information gathering is performed with the systems, and yield more evidence of their relative strengths and weaknesses.

\section{REFERENCES}

[1] Alfonseca, E., Rodríguez, P. and Pérez, D. (2007) An approach for automatic generation of adaptive hypermedia in education with multilingual knowledge discovery techniques. Computers \& Education 49(2), 495-513.

[2] Biuk-Aghai, R.P. (2006) Visualizing co-authorship networks in online Wikipedia. In Proceedings of ISCIT 2006, Bangkok, Thailand.

[3] Chen, C. and Yu, Y. (2000) Empirical studies of information visualization: a meta-analysis, In International Journal of Human Computer Studies 53(5), 851-866.

[4] Fayyad, U.M., Grinstein, G.G. and Wierse, A. (2002) Information visualization in data mining and knowledge discovery. San Francisco, CA: Morgan Kaufmann.

[5] Gawryjolek, J. and Gawrysiak, P. (2007) The Analysis and Visualization of Entries in Wiki Services. In Proceedings of WI'06, Fontainebleau, France.

[6] Green, S.J. (1998) Automated link generation: can we do better than term repetition? Computer Networks and ISDN Systems 30(1-7), 75-84.

[7] Hearst, M.A. (2009) Search User Interfaces. New York, NY: Cambridge University Press.

[8] Hirsch, C., Hosking, J. and Grundy, J. (2009a) Interactive Visualization Tools for Exploring the Semantic Graph of Large Knowledge Spaces. In Proceedings of VISSW 2009, Sanibel Island, FL.

[9] Hirsch, C., Hosking, J., Grundy, J., Chaffe, T., MacDonald, D. and Halytskyy, Y. (2009b) The Visual Wiki: A New Metaphor for Knowledge Access and Management. In Proceedings of HICSS 2009, Hawaii.

[10] Holloway, T., Božicevic, M. and Börner, K. (2007) Analyzing and Visualizing the Semantic Coverage of Wikipedia and Its Authors. In Complexity 12(3), 30-40.

[11] Kim, H. and Hirtle, S.C. (1995) Spatial metaphors and disorientation in hypertext browsing. In Behavior and Information Technology 14(4), 239-250.

[12] Kules, B. and Capra, R. (2008) Creating exploratory tasks for a faceted search interface. In Proceedings of HCIR 2008, Redmond, WA.

[13] Milne, D. and Witten, I.H. (2008) An effective, low-cost measure of semantic relatedness obtained from Wikipedia links. In Proceedings of WIKIAI'08, Chicago, IL.

[14] Munroe, R. (2007) The problem with Wikipedia. Retrieved December 2009 from http://xkcd.com/214

[15] Nunes, S. and Ribeiro, C. and David, G. (2008) Wikichanges - exposing Wikipedia revision activity. In Proceedings of WikiSym 2008, Porto, Portugal.

[16] Viégas, F.B., Wattenberg, M. and Dave, K (2004) Studying cooperation and conflict between authors with history flow visualizations. In Proceedings of CHI 2004, Vienna, Austria.

[17] Wattenberg, M., Viégas, F.B. and Hollenbach, K. (2007) Visualizing activity on Wikipedia with chromograms. In Proceedings of INTERACT 2007, Rio de Janeiro, Brazil. 